\title{
L'institution oratoire du Prince ou le savoir au service du bien dire
}

\author{
ROXANNE ROY \\ Université du Québec à Rimouski
}

Conceived somewhat in the style of the 'Mirrors of Princes' tradition composed of educational tracts addressed to future monarchs dating back to the gth century, these late sixteenth-century treatises of royal eloquence are intended to serve the Prince and edify his speech. For this reason, they invite examination as princely 'Institutions of Oratory'. The ideal portrait of the king, forever haunted by a general fear of conferring royalty upon an ass, is one of a 'learned and well-spoken' prince. Education and eloquence therefore constitute two royal virtues which allow the sovereign to distinguish himself from the people and render himself worthy of the admiration of all subjects. This primary relation between learning and eloquence taken as fundamental elements of royal power is the main concern of the present study and analysis. We shall examine the case of three 'rhetorics', composed for the use of Henry III with the intention of informing himself as a model of the 'well-spoken king'.

U n peu à la manière des miroirs des Princes, ces traités d'éducation destinés aux futurs souverains qui paraissent depuis le $\mathrm{IX}^{\mathrm{e}}$ siècle, les traités d'éloquence royale ont pour but de former le Prince et « d'instruire sa parole ${ }^{1} \gg$, nous invitant, du coup, à les envisager comme une « institution oratoire du Prince $»$. Un de leur objectif étant d'éviter au royaume de couronner un âne, le portrait idéal du roi qu'on y peint est celui d'un monarque « docte et bien disant $\gg$. L'instruction et l'éloquence seraient donc deux vertus royales qui permettraient au souverain de se distinguer du peuple et de se rendre digne de l'estime de tous. C'est ce rapport étroit entre savoir et éloquence en tant qu'assises du pouvoir royal que nous souhaitons analyser. Pour ce faire, nous nous pencherons sur le cas de trois traités de rhétorique composés à l'usage de Henri III afin de l'ériger en modèle du « roi-éloquent », soit le Projet de l'eloquence royale de Jacques Amyot, l'Avant-Discours de rhetorique, ou Traitté de l'eloquence $e^{3}$ u cardinal Du Perron et la Rhetorique francoise faicte particulierement pour le roy Henry $3^{4}$ de Germain Forget.

Nous nous intéresserons d'abord à l'éloge du savoir exposé dans les traités. Partant du principe selon lequel on ne peut être éloquent qu'à condition de bien 
maîtriser le sujet dont on parle, les traités destinés à Henri III présentent un abrégé des savoirs nécessaires au roi, et se doublent d'un enjeu rhétorique et politique. Ensuite, l'éloquence étant un art difficile qui s'apprend, on exige du Prince qu'il étudie et s'exerce. Nous verrons comment sont formulées les leçons de rhétorique dans les traités, quelle est la méthode pédagogique proposée, quelles sont les étapes que le roi doit franchir pour devenir éloquent. Puis, c'est l'éloge de l'éloquence, que l'on associe volontiers à la grandeur et à la puissance du roi puisqu'elle permet de gouverner le peuple, qui retiendra notre attention. C'est ce qui nous permettra d'envisager les traités d'éloquence royale comme relevant d'une institution oratoire du Prince, se situant au carrefour de deux genres littéraires, le miroir des Princes et l'institution oratoire.

\section{Éloquence et savoir}

Les traités d'éloquence royale présentent un véritable programme de formation destiné au Prince, qui englobe une pluralité de savoirs. Il est donc possible de penser qu'il existe une culture propre au souverain dont la fonction est de témoigner de sa grandeur, de l'élever du commun et de le rendre admirable tant par son savoir que par sa parole. En effet, les auteurs des trois traités s'entendent pour dire qu'il n'y a pas d'éloquence possible sans connaissance. « Premierement il faut faire un fonds et un magasin de toutes sortes de sciences, et acquerir la coignoissance d'infinis sujets, sans laquelle l'abondance des paroles n'est rien qu'un flux de langage inutile et ridicule : car il faut que la vraye eloquence naisse et fleurisse de la coignoissance des choses $\gg$ (TE, p. 763), écrit Du Perron sans plus de détails, soulignant au passage l'écueil que le Prince doit éviter, soit le badinage vain et ridicule, que l'on associe généralement à l'éloquence vulgaire. Les traités de Forget et d'Amyot sont beaucoup plus précis quant aux champs de savoir qui conviennent à un souverain.

L'auteur de la Rhetorique françoise ne se contente pas de présenter les différents savoirs, il le fait de manière hiérarchique. Au premier rang, on retrouve la philosophie morale (celle qui enseigne les mœurs) car elle permet au Prince de conduire sa vie et assure que le roi sera avant tout un homme de bien - or, faut-il le rappeler, l'orateur, selon Quintilien, est un « homme de bien habile dans l'art de la parole » - et l'histoire, cette fameuse « école du Prince » qui vise à former un homme juste qui saura gouverner avec prudence, magnanimité et sagesse. Pour cet auteur, l'histoire est indissociable de la chronologie et de la géographie, ce qui permet au Prince de situer les événements dans le temps et l'espace. L'élève royal apprend ainsi, pour les différents pays, leur forme de gouvernement, leurs lois, les 
mœurs et caractères des nations ; les batailles et les grands événements qui ont marqué leur histoire ; leurs principales richesses naturelles (les fleuves, les forêts, les montagnes et les plaines) ; leurs réalisations architecturales (les ports, les villes et les châteaux). Il lui suffit ensuite d'avoir une connaissance générale des arts libéraux et des sciences mécaniques.

Le programme proposé par Amyot couvre sensiblement les mêmes champs de compétences, mais il distingue les connaissances qui conviennent aux affaires communes de celles nécessaires à la conduite de l'État. Le sujet qui doit principalement retenir le Prince dans ses activités quotidiennes, lorsqu'il s'entretient avec ses courtisans par exemple, est celui des armes. Il doit apprendre tout ce qui touche à la guerre, au métier de soldat, aux diverses guerres qui ont eu lieu et aux traités de paix qui ont suivi. Ensuite, le Prince doit s'intéresser à la généalogie, à l'histoire des grandes et nobles familles du royaume. Pour Amyot, il « sied grandement à un prince d'en avoir non seulement parfaite connoissance ; $[. .$.$] mais aussi seroit très$ digne de vous qu' il ne se peut parler de guère de maisons dont vous ne sussiez à peu près l'origine et antiquité, et de plusieurs les mérites et marques les plus insignes ; comme les hautes alliances quand elles y sont ; les grands états et offices de leurs ancêtres : et spécialement leurs services signalés envers cette couronne 》 (PER, p. 66-67). Bien entendu, l'histoire demeure une discipline de choix, indispensable à tout bon Prince, d'ailleurs, elle se profilait déjà par le biais de l'étude de l'armée et de la généalogie. Ainsi que le remarque Joël Cornette, « $[s]$ cience "royale" par excellence, l'histoire permet de dessiner l'image du roi actuel à travers les générations de ses prédécesseurs ${ }^{5} \gg$. De plus, tout comme Budé et nombre de ses contemporains, on « estime qu'une bonne connaissance de l'histoire est essentielle au succès politi$q e^{6} \gg$. Amyot suggère cependant à Henri III de se consacrer à l'histoire des siècles précédents, tenant pour acquis qu'il sait l'histoire de son temps puisque c'est lui qui la fait. Le Prince apprendra ainsi l'établissement des monarchies, « les changements des règnes et empires $\gg$, « les fautes ou les sages conduites des gouvernements », «les maladies et mortalités des états souverains », « les beaux moyens et remèdes par où les Royaumes ont été conservés et accrus », « les vertueuses et prudentes actions » des souverains, « les guerres sagement entreprises », « les moyens par lesquels les Rois ont été chéris ou haïs de leurs peuples $\gg$, , les belles lois établies par les bons Princes et le grand ordre tenu par eux $\gg$ (PER, p. 68). La chasse, les bâtiments, les pierres précieuses et autres sujets semblables peuvent aussi meubler la conversation.

Curieusement, Amyot est peu loquace quand il s'agit de la matière à étudier pour la conduite de l'État. Tout au plus est-il question des affaires (politiques) du 
temps, sujet qu' il convient d'adapter au rang de l'interlocuteur et aux circonstances. Il se contente de formuler un principe où profit et diversité sont les mots d'ordre : « Puisque le but principal où tend un bon prince n'est que la commodité de ses sujets et le bien du Royaume, il semble que le Roi parle pour son profit et pour celui d'autrui tout ensemble quand il est question de ses affaires, dont nous pourrons connoître la diversité par la diversité des personnes » (PER, p. 74).

Le programme peut paraître vaste et Amyot réfute à l'avance les objections qu'on pourrait lui faire, réaffirmant du coup l'importance de « beaucoup sçavoir pour bien dire $\gg:$ « c'est que le premier et principal point de l'éloquence gît à ne parler d'aucune chose dont on n'ait bonne intelligence; et ceux qui ont enseignél'art de bien dire ne l'avoir autrement formé qu'avec la connoissance des belles sciences, sans lesquelles ce qu'on appelleroit éloquence ne seroit à la vérité qu'une baverie indiscrète et ignorante $\gg(P E R$, p. 71-72).

Les traités d'éloquence royale sont donc très proches de l'Institution oratoire car l'enseignement qu' ils préconisent est avant tout lié à l'invention, cette première partie de la rhétorique qui consiste à trouver la matière et le sujet dont il convient de parler. Les remarques de Jean Cousin vont dans ce sens :

Pour Quintilien, la matière [de la rhétorique] est plus vaste : ce sont tous les sujets, y compris la philosophie, sur lesquels elle sera appelée à parler. Cela implique donc une vaste culture, et l'on comprend que, dès l'école du grammairien, l'auteur de l'Institution oratoire ait songé à ouvrir l'esprit de l'élève à une culture qu'étendra et qu'appropriera le rhéteur et que développeront l'expérience et la vie. Programme ambitieux, programme immense qu'explique et justifie l'ardente foi de Quintilien dans la rhétorique ${ }^{7}$.

Or, si l'étude de l'histoire, de la philosophie morale ou de la géographie doit former l'esprit du Prince et lui inculquer des notions utiles pour bien gouverner, elle doit aussi lui permettre de « parler mieux que nul autre, soit pour l'ornement du langage, pour la force des raisons, ou pour l'autorité des exemples $\gg(P E R$, p. 64). Plus les connaissances du Prince sont étendues et solides, mieux il sait prouver ce qu'il dit, que ce soit à l'aide d'exemples, de sentences ou de lieux communs. Le savoir acquis améliore la qualité de l'argumentation du Prince et sa force de persuasion, et en maîtrisant ainsi la parole et le discours, il parvient à surpasser tous ses sujets.

Les traités d'éloquence à l'étude rejoignent le miroir du Prince, car le savoir qu' ils diffusent doit aussi (et peut-être surtout) permettre au Prince de bien diriger ses sujets, que ce soit par la conduite des affaires de l'État, de l'armée, des lois ou de la justice. La formation n'est certes pas dénuée d'intérêt, car elle permet au Prince de s'assurer de l'attachement et de la fidélité de ses sujets, de gagner leur 
estime, voire leur affection. C'est d'ailleurs ce que dit explicitement Amyot quand il recommande au Prince d'être féru en histoire des nobles familles du Royaume : «De quoi vous sçaurez si bien user que par ce moyen vous retiendrez en office beaucoup d'hommes de votre noblesse, leur rappelant les beaux faits et gestes de leurs prédécesseurs » (PER, p. 67). L'éloge du savoir que l'on trouve dans ces traités est donc au fondement de la légitimité politique. Tout comme le faisait déjà Budé dans L'institution $d u$ Prince, ils attachent une extrême importance à l'éducation du Prince, « convaincu $[\mathrm{s}]$ que le roi est seul dépositaire de l'autorité royale ${ }^{8} \gg$. Ils valorisent la culture au point d'en faire une vertu royale, qui est propre au souverain et qui le rend digne de gouverner.

\section{Éloquence et pédagogie}

L'éloquence, selon Quintilien, est un art qui nécessite un apprentissage devant se faire avec ordre et méthode. Pour Du Perron, c'est justement la difficulté liée à cet apprentissage qui rend l'éloquence digne d'être étudiée par un Prince et qui explique le petit nombre «d'excellents Orateurs, qui ont esté remarquez par tous les siecles $\gg(T E$, p. 762). Puisqu' il y a peu d'élus, le Prince a tout avantage à se consacrer avec sérieux et application à cette étude. Les auteurs des traités d'éloquence royale incitent donc Henri III à devenir un monarque éloquent, car ils y voient un moyen assuré pour lui de se distinguer et d'être admiré par la postérité.

Lorsqu'il est question de pédagogie, les traités d'éloquence royale s'inscrivent dans la tradition de l'Institution oratoire. D'ailleurs, pour Théodore Froment, $\ll$ [1]'Institution oratoire n'est pas seulement le cours de rhétorique le plus complet que nous ait laissé l'Antiquité : c'est en même temps un traité d'éducation, un véritable manuel de pédagogie ${ }^{9} \gg$. Les traités proposent une méthode adaptée particulièrement aux besoins du Prince. D’abord, puisqu'il faut de vastes connaissances pour être éloquent, la lecture des bons auteurs s'impose. Amyot reconnaît l'importance de $\ll$ la lecture des bons livres » (PER, p. 69), mais il ne lui semble pas nécessaire de préciser lesquels. Il recommande par contre au Prince de lire un manuel d'histoire, un «abrégé composé d'art et de méthode » (PER, p. 68), car « ces choses écrites de bonne plume et digérées par ordre pour votre mémoire tant heureuse et présente, accompagneroient votre parler ordinaire de grâces infinies quand il vous plairoit l'orner et parer de si excellentes fleurs $\gg$ (PER, p. 69). Les avantages de la lecture sont donc multiples. Non seulement elle accroît les connaissances et forme les idées, mais elle permet de corriger le style puisqu'elle fournit des exemples d'ornements et de figures qui permettront à son tour au Prince de fleurir son discours. La lecture 
permet également d'exercer la mémoire. Amyot, tout en complimentant le Prince sur sa mémoire « dont un chacun admire l'excellence », en souligne l'importance « pour comprendre et retenir toutes sciences dignes d'un Roi » (PER, p. 55). Or, la mémoire est essentielle à la pratique de l'éloquence et constitue la quatrième partie de la rhétorique. Du Perron écrit justement à ce sujet : « quant à la memoire chacun peut penser combien les autres perfections de l'orateur luy seront inutiles, si ceste-cy n'est employée pour les conserver seurement et fidellement » (TE, p. 764).

L'auteur de la Rhetorique francoise précise davantage le programme de lecture destiné au Prince. Puisque la connaissance de la philosophie morale « s'acquiert par une lecture méthodique $\gg(R F$, p. 15), il recommande les ouvrages de Platon, Aristote, Plutarque, Cicéron, Sénèque, Pline et autres. À propos des manuels d'histoire, il précise que les auteurs doivent être crédibles, de véritables chercheurs spécialistes du sujet dont ils traitent. Les travaux de Pomponius Mela, sans doute le premier géographe romain, conviennent pour apprendre la géographie et la chronologie. Les topiques d'Aristote, de Cicéron et l'Institution oratoire sont les ouvrages de référence pour apprendre les lieux communs. Tout comme Quintilien qui souligne la nécessité de choisir le meilleur des maîtres de rhétorique et exige de lui qu'il soit un excellent pédagogue, Germain Forget insiste particulièrement sur l'importance du rôle du précepteur dans la formation du Prince. Il lui faut d'abord traduire en français le livre d'un bon auteur (puisque le roi connaît mal le latin), en faire la lecture au Prince, rapporter en une sorte de synthèse l'avis d'autres auteurs sur la même question, expliquer et éclaircir le sujet dont il est traité, donner différents exemples au besoin. La leçon magistrale est suivie d'exercices qui visent à mettre en pratique l'enseignement reçu. Voici comment il présente cette méthode :

Quelque docte personnage traduira les offices de Ciceron en françois et en fera lecture d'un chapitre pour chacun jour, puis l'explicra a Votre Majesté, discourant sur le subiect d'iceluy, raportant tout ce que les autheurs Grecs et Ronmains en auront escript, puis l'eclaircira des exemples de l'antiquité tiré des histoires et fables enciennes. V. D. Maiesté fera semblable discours apres redigé par escrip, ou elle observera toutes les prescriptions (preceptes) et loys de Rhetorique, et le poura reciter par cœur devant son homme lettré qui en donnera son advis ; et par inssy sera le moyen d'aprendre, par maniere d'esbat et d'exercice, la philosophie, l'hystoire, les fables conjointes avec l'eloquence, et reduire le tout en pratique. (RF, p. 16-17)

De la même manière, pour Du Perron, il ne suffit pas d'acquérir des connaissances et des préceptes pour être éloquent, le Prince doit aussi faire des exercices, passant ainsi de la théorie à la pratique. La méthode qu'il préconise s'apparente à celle de Quintilien pour qui il importe d'unir la théorie à la pratique. Ce dernier 
expose un programme en trois temps où les exercices proposés vont du plus simple au plus complexe. L'élève doit débuter par la narration (celle de la fabula, de l'argumentum et de l'historia), puis réfuter ou confirmer ce qu'apportent ces narrations et, enfin, s'adonner à une variété d'exercices qui vont de l'éloge au blâme, en passant par la comparaison et le développement des lieux communs, les thèses, l'examen de la uoluntas, la discussion des témoignages, l'éloge ou la critique des lois ${ }^{10}$. Pour Du Perron, cet apprentissage se fait graduellement, c'est-à-dire qu'il doit d'abord imiter les bons auteurs, puis inventer ses propres discours, en prenant soin de respecter les règles de l'éloquence : « il y a encore un certain moyen entre l'art et l'usage, qui est l'imitation, c'est à dire, que devant que s'exercer luy-mesme en œuvres de son invention, il est besoin qu'il pratique premierement les reigles de l'art, en imitant les labeurs des bons ; et que ne pouvant encores cheminer de luy-mesme, il se soustienne sur leurs exemples » (TE, p. 768). L'apprentissage de l'éloquence tiendrait donc en quatre principes : « en la bonté de la nature, en la cognoissance de l'art et des preceptes, en l'imitation des excellents ouvriers, et en l'usage ou l'exercice » (TE, p. 769). L'importance accordée à l'imitation le distingue de Quintilien pour qui l'habileté et la maîtrise de la parole dépendent principalement « de la nature, de l'art, de l'exercice ».

Sans doute est-ce par leur usage de l'histoire que les traités d'éloquence royale suivent au plus près le modèle des miroirs des Princes, qui «sont tout à la fois des manuels utiles au gouvernant, des guides moraux soulignant les vertus qui leur sont nécessaires (prudence, justice, magnanimité) et de véritables traités politiques ${ }^{11} \gg$. En effet, la forme même des traités d'éloquence s'apparente à ces recueils d'exempla qui renvoient au Prince l'image d'un roi idéal et l'incitent à suivre cette voie. Les préceptes enseignés par les maîtres d'éloquence sont presque toujours accompagnés d'exemples de rois, de princes, d'empereurs ou de généraux tirés de l'histoire antique ou contemporaine $\mathrm{e}^{12}$. Au chapitre $\mathrm{V}$ de son Projet de l'eloquence royale, Amyot affirme que la fine moquerie convient mieux aux propos du Prince, qu'ils doivent «plutôt chatouiller que piquer aigrement » (PER, p. 58). Pour ce faire, il prend pour exemple un mot d'esprit du roi Louis XI. Il formule la leçon en ces termes : « Il peut néanmoins toucher quelquefois jusqu'au vif, sous paroles douces et couvertes, ainsi que le fit le Roi Louis onzième » (PER, p. 58). Le roi, assommé par la présentation pompeuse d'un certain évêque, répondit : « Où il y a tant de titres, [ ... ] il y a peu de lettres $\gg$ (PER, p. 59). Plus loin, Amyot poursuit en mettant le Prince en garde contre le mauvais usage de la raillerie :

il faut éviter sur tout en une risée qu'elle ne se puisse retourner contre son auteur ; ce qui advint jadis à l'empereur Auguste. Quelqu'un l'avertit qu'il étoit venu un 
étranger à Rome qui lui ressembloit parfaitement. Il le voulut voir : et se pensant jouer de lui, il lui demanda si jamais sa mère n'étoit venue à Rome. L'étranger lui répondit promptement : Non pas ma mère que je sçache : mais mon père y est venu plusieurs fois. (PER, p. 60)

Les auteurs des traités pratiquent donc une pédagogie par l'exemple où il s'agit d'imiter les conduites vertueuses et de fuir celles qui sont répréhensibles. Or, comme le remarque Christian Jouhaud, l'histoire se présentant comme une « école de vertu » qui «montre les bons et les mauvais exemples ${ }^{13}$ », il n'est pas étonnant que les traités d'éloquence royale lui accordent une si grande importance. Non seulement les exemples historiques agissent à titre d'arguments d'autorité, mais ils participent à l'enrichissement des connaissances historiques du Prince.

Notons également que le Prince, par un jeu de dédoublement, renvoie à son tour une image parfaite aux nobles qui l'entourent quand il aborde le sujet des grandes familles royales. Il leur présente en quelque sorte un « miroir du courtisan », visant à les éduquer selon ce même principe d'émulation. La leçon ne saurait être plus claire : les nobles éprouveront du bonheur et de la satisfaction « quand par vous-même [le Prince] ils entendront remémorer ce qui les doit inciter à la vertu et honnête imitation de leurs plus proches $\gg(P E R$, p. 67). Lire, discuter, imiter, puis écrire sont donc au fondement de l'éducation du prince, qu'il s'agisse d'apprendre à être éloquent ou à gouverner.

\section{Éloquence et politique}

L'enseignement de l'éloquence répond à une volonté politique, ainsi que le souligne Philippe-Joseph Salazar ${ }^{14}$, car la parole du Prince ne doit pas seulement être vraie et belle, elle doit aussi être efficace. Le cours d'éloquence de ces trois éducateurs étant destiné à Henri III, il est donc conçu de manière à répondre aux fonctions $\mathrm{du}$ Prince et à le former au «métier de roi $\gg$.

Voulant susciter l'intérêt du Prince pour l'étude de l'éloquence, Du Perron débute son discours en insistant sur son utilité pour les diverses formes de gouvernement. C'est parce que l'éloquence est un instrument qui permet de toucher et de persuader le peuple, qu'elle permet aux dirigeants de mieux gouverner. Son avantage est d'ailleurs loin d'être négligeable : « car c'est elle qui meine les assemblées des hommes toutes entieres par la parole, se rend maistresse de leurs affections, tourne leurs volontez où bon luy semble, et les retire de là où il ne luy plaist pas qu'elles soient inclinées $\gg$ (TE, p. 759). On comprend facilement pourquoi le Prince a tout intérêt à devenir un «monarque éloquent ». S'il est vrai que l'éloquence a régné en 
maître dans les Républiques et les gouvernements populaires, elle convient « aussi aux Royaumes et Monarchies, à cause que les deliberations y dépendent de la volonté d'un seul, qui est ordinairement plus instruict et preparé de l'estat des choses que ne sçauroit estre une multitude grossiere et ignorante $\gg$ (TE, p. 760). Du Perron esquisse au passage le portrait du Souverain modèle qui doit d'abord être un homme instruit et éloquent afin de conduire son peuple avec sagesse et raison. Il insiste ensuite sur l'utilité de l'éloquence dans le maniement des affaires, précisant les circonstances dans lesquelles le Prince peut en faire bon usage :

Il y a les ambassades vers les Princes estrangers, il y a les propositions au Conseil d'Estat, il y a les relations, quand on vient rendre compte de quelque longue commission qu'on exerce; il y a les harangues qu'on peut prononcer aux Parlements, aux principaux sieges des Provinces, lors qu'on y prend possession de quelque gouvernement ou de quelque authorité ; il y a celles qu'on peut faire aux corps de villes, et aux assemblées du peuple, et principalement en une saison telle qu'est la nostre, c'est à dire, durant les guerres civiles, menées par des pretextes populaires, comme pour la Religion. (TE, p. 760)

Pour Amyot, l'éloquence doit être estimée du roi car elle lui est « grandement recommandable, profitable, voire nécessaire ; et [...] s'il en sçait user dextrement et à point, il en établira, maintiendra, et augmentera son état, autant ou plus que par nul autre moyen dont les Royaumes et grandes seigneuries s'entretiennent » (PER, p. 45-46). La parole du Prince est donc un instrument de puissance qui assure sa grandeur et son autorité. Plus encore, l'éloquence, par sa force de persuasion, permet de gagner à sa cause les peuples et les cités, aussi sûrement (sinon plus) que les armes. Amyot a à cœur de montrer au souverain, par une série d'exemples historiques (Pyrrhus, Jules César, Mithridate, le Grand Roi Perse), « combien cette science peut servir aux Princes ». Pacifiste en quelque sorte, il prône la supériorité de la parole sur la force physique : « Joint qu'il survient des saisons où la force n'a lieu aucun, et la persuasion tout pouvoir $\gg(P E R$, p. 48 ). Puis, il termine son plaidoyer en faveur de l'éloquence par cette maxime : «si l'éloquence est reine de toutes choses, [ ... ] il n'y a Roi, tant soit grand et puissant, qui ne doive désirer de l'avoir pour sa compagne $\gg$ (PER, p. 49).

Lorsqu'ils enseignent comment rédiger un discours (une harangue ou une oraison) et qu'ils expliquent quelles en sont les quatre parties, les auteurs des traités d'éloquence royale prennent soin d'adapter leurs préceptes aux circonstances où le souverain prend la parole. L'exorde, en particulier, est longuement développé, ce qui s'explique par le fait que le Prince doit alors capter l'attention et la bienveillance de son auditoire, le disposer à bien écouter et recevoir ce dont il veut les entretenir. 
On trouve donc une série de conseils pratiques, à l'usage exclusif du Prince, qui doivent lui permettre de devenir un excellent orateur. Selon Amyot, le Prince doit respecter quatre éléments-clés : « il suffit à un Roi, à qui son autorité donne assez d'audience, de sobrement louer les auditeurs $\gg$, de « parler de soi sans arrogance et avec dignité », de préciser que « la chose dont il parlera est profitable au public » et de prendre garde à ce que la longueur de cet exorde soit d'une longueur proportionnelle à l'ensemble du discours.

Germain Forget, dans la Rhetorique françoise, n'est pas avare de conseils. Les préceptes généraux au sujet de la captatio benevolentiae sont sensiblement les mêmes : le Prince doit montrer que c'est le bien commun et l'honneur du royaume qui l'incitent à prendre la parole; que le propos dont il veut les persuader est juste et bon ${ }^{15}$, puis il peut souligner ses propres qualités afin de montrer qu'il est digne d'estime et de confiance. Le maître d'éloquence fournit ensuite au Prince de nombreux exemples qui tiennent compte de la qualité des ses interlocuteurs et de leur sensibilité, et c'est en cela qu'il se distingue le plus nettement de l'Institution oratoire. Quand il s'agit de capter la bienveillance des parlementaires, députés d'état ou autres personnages de marque, il ne faut pas hésiter à vanter leur mérite et leur vertu, voire à les flatter : «ce parlement lâ a tousjours flory en hommes tres vertueux et bien dignes de leur charge ; que c'est la vray siege et habitacle de la justice ; que les predecesseurs Roys ont tousjours eu beaucoup de respect a un tel cors et college, leur communiquant toutes les importantes affaires du royaulme et prenant leur advis en toutes choses $\gg$ (RF, p. 44). Il convient de louer « la fidelité et l'obeissance de nos sujets $\gg$ afin de les inciter plus facilement à rendre le service qu'on leur demande. Ainsi, s'il s'adresse à des ambassadeurs de provinces, à des princes ou à des rois étrangers : « nous dirons combien ces deux nations ont tousjours esté conjointes d'amitié ; nous en raporterons et reciterons quelque specialité ou particularité, comme elles se sont autrefois secoureus de forces et de conseil $\gg(R F$, p. 44).

Il est à remarquer que si les traités d'éloquence n'oublient pas d'aborder la question de l'élocution (figures) et de l'action (prononciation, voix, corps et gestes), ces deux parties ne semblent toutefois pas supposer un enseignement propre au Prince. Les préceptes sont valables pour tout bon orateur, ainsi que le montre cet extrait tiré du traité de Du Perron au sujet de l'élocution : «l'Orateur doit estre riche et abondant de beaux mots, de belles constructions, de belles figures, pour revestir et orner les conceptions de son esprit » (TE, p. 763). De même, à propos de l'action, il écrit que le discours, pour être convaincant, doit « estre accompagné d'une belle et agréable action, qui consiste en une douce et harmonieuse voix, en un plaisant et gracieux aspect du visage, en une bienseance de geste et mouvements de 
tout le corps ; laquelle partie seule, est de telle importance, que sans elle, l'oraison demeure froide, stupide et inanimée $\gg$ (TE, p. 764). C'est ce qui nous permet de croire que le propre de l'éloquence royale réside dans l'invention, l'argumentation (la disposition) et la mémoire. Ce constat rejoint les propos de Philippe-Joseph Salazar pour qui « [i]l n'y a qu'un pas à franchir pour affirmer que l'élocution, la prononciation et l'action relèvent du logos extérieur et que la distinction entre la parole intérieure, l'entendement, et la parole proférée, la "parole" dit Amyot, recouvre en fait la première distinction entre la gravité de l'éloquence royale et le babil de l'éloquence vulgaire ${ }^{16} \gg$.

Si L'USAGE DE LA PAROLE distingue l'homme de la bête (ce que n'ont de cesse de répéter les traités de rhétorique $\left.{ }^{17}\right)$, l'éloquence royale, composée de l'invention, de la disposition et de la mémoire, doit distinguer le Prince de son peuple. Ainsi, l'éloquence serait une discipline fondamentale qui joue un rôle de premier plan dans la formation du souverain. Elle a pour avantage d'exercer l'esprit, d'affermir le jugement et la mémoire du Prince, qualités qui témoignent du caractère d'élection du souverain. L'ensemble des connaissances qu'elle convoque témoigne de la supériorité du Prince face à son peuple, il peut ainsi asseoir son autorité, alors que la maîtrise de la parole éloquente lui permet de mieux gouverner ses sujets. Cette lecture croisée des textes nous montre, finalement, qu'en choisissant Henri III comme destinataire, les auteurs des traités d'éloquence royale ne pouvaient qu'orienter leur enseignement vers une finalité politique, tenant compte de la fonction et des devoirs inhérents au métier de roi, nous permettant, de ce fait, de les envisager comme des « institutions oratoires du Prince ».

\section{Notes}

1. Philippe-Joseph Salazar, « Préface », dans Jacques Amyot, Projet d'éloquence royale, Paris, Les Belles Lettres, coll. Le corps éloquent, 1992, p. 35.

2. Jacques Amyot, Projet d'éloquence royale, Paris, Les Belles Lettres, coll. « Le corps éloquent $\gg, 1992$. Désormais, les références à cet ouvrage seront désignées à l'aide du sigle $P E R$, suivi de la page, et placées entre parenthèses dans le texte.

3. Jacques Davy Du Perron, « Avant-discours de rhetorique, ou Traitté de l'eloquence », dans Euvres diverses, Paris, Pierre Chaudiere, 1633, p. 759-770. Désormais, les références à cet ouvrage seront désignées à l'aide du sigle $T E$, suivi de la page, et placées entre parenthèses dans le texte. 
4. Rhetorique francoise faicte particulierement pour le roy Henry 3 dans Precetti di rettorica scritti per Enrico III re di Francia, édition de Giulio Camus, Modène, Antica tipografia Soliani, 1887. Désormais, les références à cet ouvrage seront désignées à l'aide du sigle $R F$, suivi de la page, et placées entre parenthèses dans le texte.

5. Joël Cornette, «Le savoir des enfants du roi sous la monarchie absolue $\gg$, dans Ran Halévi (sous la dir. de), Le savoir du prince du Moyen Âge aux Lumières, Paris, Fayard, 2002, p. 131.

6. Robert J. Knecht, « François I ${ }^{\text {er }}$ et le "Miroir des Princes” », dans Ran Halévi (sous la dir. de), Le savoir du prince du Moyen Âge aux Lumières, ouvr. cité, p. 99

7. Jean Cousin, « Notice », dans Quintilien, Institution oratoire, Paris, Les Belles lettres, collection des Universités de France, 1976, tome II, livres II-III, p. 23.

8. Robert J. Knecht, « François I ${ }^{\text {er }}$ et le "Miroir des Princes" », art. cité, p. 101.

9. Théodore Froment, « Quintilien », dicoFB, document.php?id=3480. Site $:<\mathrm{http}: / /$ www.inrp.fr/edition-electronique/lodel/dictionnaire-ferdinand-buisson/ document.php?id=3480> (page consultée le 14 juillet 2007).

10. Voir, à ce propos, Jean Cousin, « Notice », dans Quintilien, Institution oratoire, ouvr. cité, p. 5-6.

11. Source : <http://gallica.bnf.fr/themes/PolXVI1.htm> (page consultée le 14 juillet 2007).

12. On retrouve au sein de cette galerie d'hommes illustres le fameux Hercule gaulois aussi bien que Marc Antoine, Pisistrate, Cléomène, Périclès, Tibère, Auguste, Cyrus, sans oublier François I ${ }^{\text {er }}$ et Henri III lui-même.

13. Christian Jouhaud, « Une "religion du roi" équivoque. Le prince de Jean Louis Guez de Blazac », dans Ran Halévi (sous la dir. de), Le savoir du prince du Moyen Âge aux Lumières, ouvr. cité, p. 183.

14. Philippe-Joseph Salazar, « Préface », dans Jacques Amyot, Projet d'éloquence royale, ouvr. cité, p. 13-14.

15. Bien que cette remarque tienne compte de la qualité du Prince, elle ne diffère pas véritablement des propos de Quintilien. Selon lui, l'orateur désirant capter l'attention de l'auditoire doit dire que le sujet dont il souhaite les entretenir est important, vrai et en accord avec la morale.

16. Philippe-Joseph Salazar, « Préface », dans Jacques Amyot, Projet d'éloquence royale, ouvr. cité, p. 20-21.

17. «Or puis que nous avons, comme par preciput, sur tous animaux le bien de pouvoir deviser et discourir entre nous, et découvrir nos pensées les uns aux autres par l'usage de la parole, certes nous en devons faire beaucoup d'estime, et nous faut mettre peine d'acquérir encore cet avantage, que la même chose qui avant toutes autres nous fait surmonter les bêtes, soit celle aussi par la vigueur de laquelle nous surmontions les autres hommes. $\gg$ (PER, p. 44). Voir aussi TE, p. 761. 